PrePared For SUbMission to JINST

inStR2o: Instrumentation for Colliding Beam Physics

24-28 FEBRUARY 2020,

NovosiBIRSK, RussiA

\title{
Beam test characterisation of a Plastic Scintillator Prototype for the space-based cosmic ray experiment HERD
}

\author{
P.W. Cattaneo, ${ }^{a}{ }^{1}$ M. Pullia, ${ }^{b}$ M.C. Prata, ${ }^{a}$ A. Rappoldi ${ }^{a}$ and M. Rossella ${ }^{a}$ \\ ${ }^{a}$ INFN Pavia, Via Bassi 6, Pavia, Italy \\ ${ }^{b}$ CNAO, Str. Campeggi, 53, Pavia, Italy \\ E-mail: paolo.cattaneo@pv.infn.it
}

\begin{abstract}
The High Energy cosmic-Radiation Detector (HERD) facility is planned to go onboard China's Space Station, planned to be operational starting in around 2025 for about 10 years. The main scientific objectives of HERD are the search for signals of dark matter annihilation products, precise cosmic electron/positron spectrum and measurements of anisotropy up to $10 \mathrm{TeV}$, precise cosmic ray spectrum and composition measurements up to the knee energy ( $1 \mathrm{PeV})$, and high energy $\gamma$-ray monitoring and survey. HERD consists of a 3D cubic crystals calorimeter (CALO) surrounded by microstrip silicon trackers (STKs) and scintillating fiber trackers (FIT) and by a Plastic Scintillator Detector (PSD) for $\gamma$-ray veto and ion charge measurement. A PSD prototype consisting of a scintillator tile readout by two arrays of SiPMs on opposite sides has been tested with proton and $\mathrm{C}$ ion beam at the CNAO (Centro Nazionale Adroterapia Oncologica) in Pavia, (Italy). Preliminary results on charge resolution are presented.
\end{abstract}

KeYwords: Scintillators, Photon detectors for UV, visible and IR photons (SiPM), dE/dx detectors

\footnotetext{
${ }^{1}$ Corresponding author.
} 


\section{Contents}

1 The HERD experiment 1

2 The accelerator facility 1

3 The device under test 3

4 Results 4

5 Conclusions 5

\section{The HERD experiment}

The High Energy cosmic-Radiation Detection (HERD) facility will be one of the space astronomy payloads on board the future Chinese space station. The goal of HERD is the direct detection of cosmic rays at the knee region ( $1 \mathrm{PeV}$ ), with a detector able to measure electrons, photons and nuclei with high energy resolution (1\% for electrons and photons at $200 \mathrm{GeV}$ and $20 \%$ for nuclei at $100 \mathrm{GeV}-\mathrm{PeV}$ ), a geometrical factor ten times larger than the one of present generation missions ( $>2 \mathrm{~m}^{2} \mathrm{sr}$ ), and life-time longer than ten years [1]. The primary objectives of HERD are the indirect search for dark matter particles and the precise measurement of energy distribution and composition of cosmic rays from $30 \mathrm{GeV}$ up to a few $\mathrm{PeV}$, determining the origin of the knee structure of the spectrum. Furthermore, HERD will monitor the high energy gamma-ray sky from $\sim 500 \mathrm{MeV}$, observing gamma-ray bursts, active galactic nuclei, galactic microquasars, etc. HERD will be composed of a homogeneous calorimeter, surrounded by a particle tracker and a plastic scintillator detector (PSD) as shown in Fig. 1. The PSD consists of one or two layers of plastic scintillators surrounding the whole detector segmented in bars or tiles readout by SiPMs. Its goal is to provide trigger and veto information and to measure the atomic number $\mathrm{Z}$ of the ions through their energy loss.

The two mechanical solutions under investigations are sketched in Fig. 2 where the PSD can be segmented in tiles or bars. In the following we present the test of a prototype of the tile solution.

\section{The accelerator facility}

The CNAO ${ }^{1}$ [4] is a accelerator facility dedicated a hadron therapy to treat cancer, see Fig.3. The beams available at CNAO are protons with kinetic energy in the continuous range $60-250 \mathrm{MeV}$ and $\mathrm{C}$ ions with kinetic energy in the continuous range $120-400 \mathrm{MeV} / \mathrm{u}$. The rationale behind these ranges is that hadron therapy is particularly effective because of the large energy release at the Bragg peak for hadrons close to their stopping points.

\footnotetext{
${ }^{1}$ Centro Nazionale Adroterapia Oncologica, Pavia, Italy
} 


\section{HERD detector and requirements}

\begin{tabular}{|c|c|}
\hline$\sigma_{\theta}(\mathrm{y})$ & $0.1^{\circ} @ 10 \mathrm{GeV}$ \\
\hline $\mathrm{Z}$ & $1-26$ \\
\hline$\sigma_{z}$ & $0.1-0.15 \mathrm{e}$ \\
\hline $\mathrm{e} / \mathrm{p}$ & $10^{6}$ \\
\hline
\end{tabular}

\begin{tabular}{|c|}
\hline Energy \\
Range \\
\hline Energy \\
resolution \\
\hline $\begin{array}{c}\text { Effective } \\
\text { GF }\end{array}$ \\
\hline
\end{tabular}

\begin{tabular}{|c|c|}
\hline $\mathrm{e}$ & $\mathrm{p}$, nuclei \\
\hline $10 \mathrm{GeV}-$ & $30 \mathrm{GeV}-$ \\
$100 \mathrm{TeV}$ & $3 \mathrm{PeV}$ \\
$1 \% @ 200$ & $20 \% @ 100$ \\
$\mathrm{GeV}$ & $\mathrm{GeV}-1 \mathrm{PeV}$ \\
\hline $3 \mathrm{~m}^{2} \mathrm{sr}$ & $>2 \mathrm{~m}^{2} \mathrm{sr}$ \\
$@ 200 \mathrm{GeV}$ & $@ 100 \mathrm{TeV}$ \\
\hline
\end{tabular}
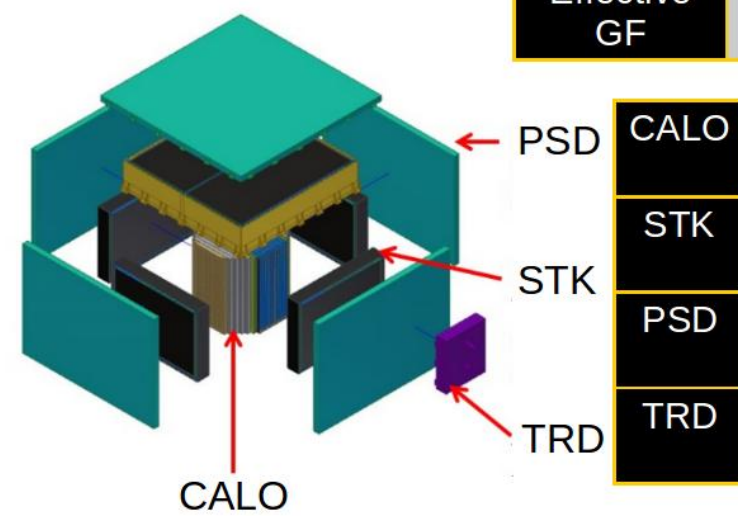

Energy Reconstruction e/p Discrimination

Trajectory Reconstruction Charge Identification

Charge Reconstruction LE y Identification

Calibration of CALO response for $\mathrm{TeV}$ proton

Figure 1. A sketch of the HERD detector.

\section{HERD Plastic Scintillator Detector (PSD)}

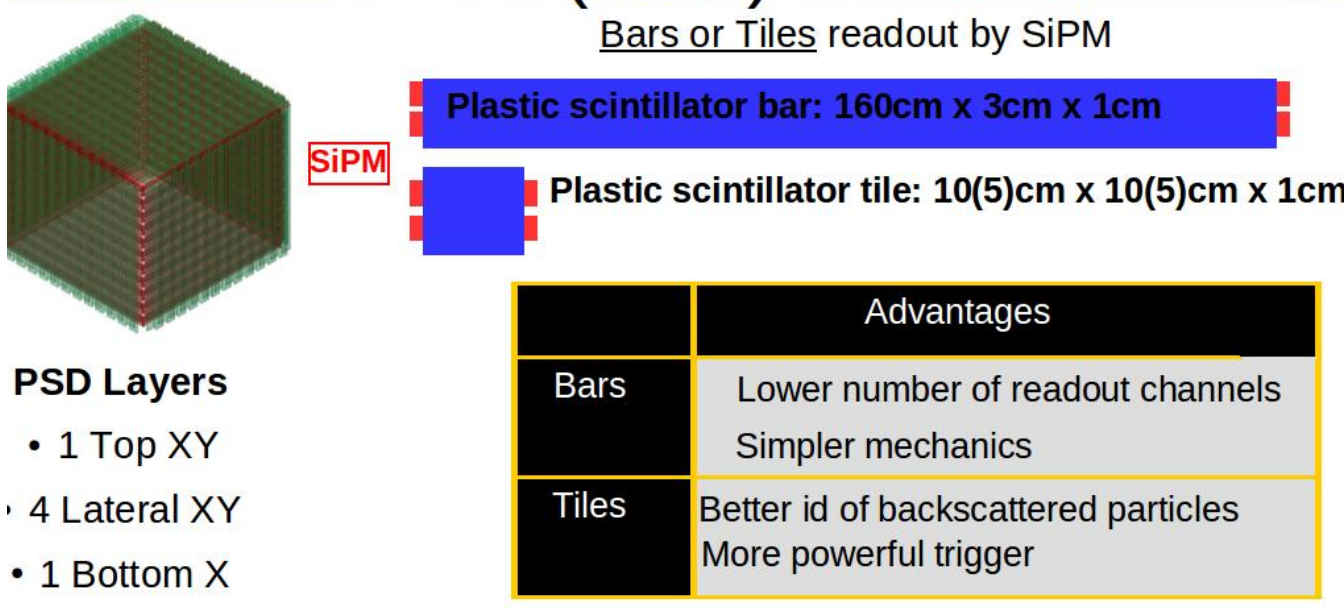

Figure 2. A sketch of the two possible solutions for the HERD PSD. 
The particle rate available is up to $10^{10} \mathrm{p} / \mathrm{s}$ or $4 \times 10^{8} \mathrm{C} / \mathrm{s}$ and can be scaled down almost arbitrarily with appropriate support from the accelerator operators. The beam spot is Gaussian in shape of size $\sigma_{x, y}=0.2-0.8 \mathrm{~cm}$ depending on the energy and ion type.

In the following measurements the beam rate was tuned to a rate sufficiently low to have a negligible pileup.
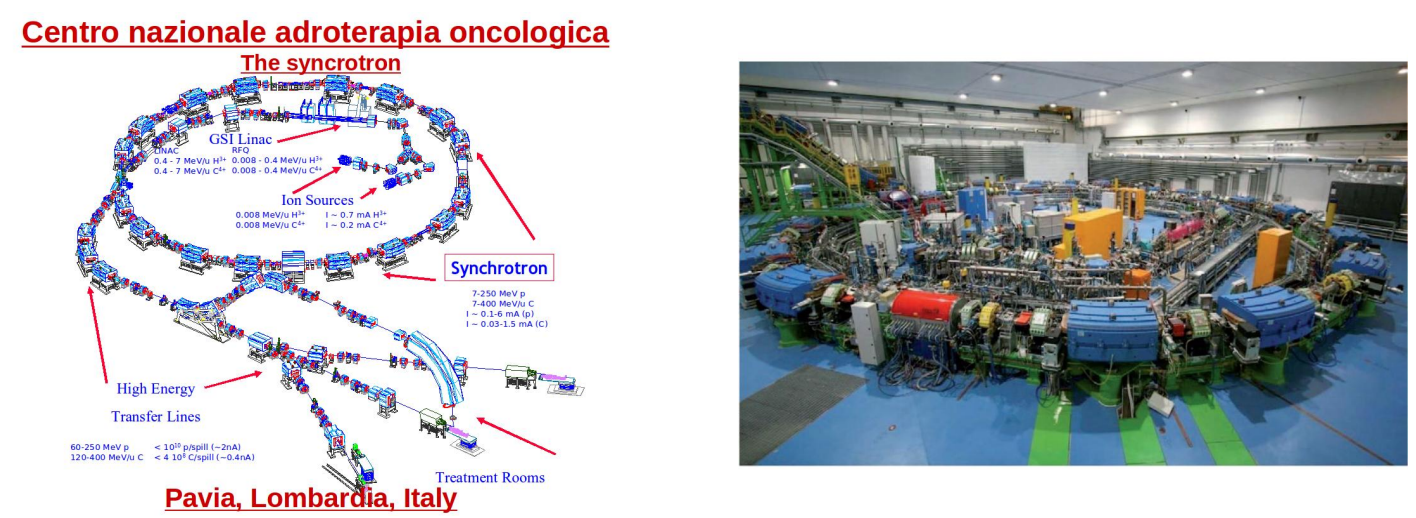

Figure 3. A sketch of the CNAO synchrotron (left). A view of the CNAO synchrotron (right).

Table 1. Ion types used in the test with beam kinetic energies per nucleon $\mathrm{E}_{\mathrm{k}} / \mathrm{A}, \beta$ and $(Z / \beta)^{2}$ (see text).

\begin{tabular}{|l|c|c|c|c|c|c|c|c|c|}
\hline Beam type & \multicolumn{5}{|c|}{$\mathrm{p}$} & \multicolumn{5}{c|}{$\mathrm{C}$} \\
\hline $\mathrm{E}_{\mathrm{k}} / \mathrm{A}(\mathrm{MeV})$ & 70 & 120 & 170 & 226 & 115 & 190 & 260 & 330 & 400 \\
$\beta$ & 0.366 & 0.462 & 0.532 & 0.592 & 0.454 & 0.555 & 0.622 & 0.672 & 0.713 \\
$(Z / \beta)^{2}$ & 7.46 & 4.68 & 3.53 & 2.85 & 174.6 & 116.9 & 93.0 & 79.7 & 70.8 \\
\hline
\end{tabular}

We exploited the full energy range for both ions; the energy values selected are shown in Table 1.

The energy loss in the PSD prototype of ions with the energies in Table 1 is given by the Bethe-Bloch formula that describes the energy loss in $\mathrm{MeV}$ per length times density [3]

$$
\frac{d E}{d(x \rho)} \sim(Z / \beta)^{2} 2.0 \mathrm{MeV} /\left(\mathrm{g} / \mathrm{cm}^{2}\right)
$$

where $\mathrm{Z}$ is the atomic number of the impinging ion and $\beta$ its velocity. The energy loss is proportional to $(Z / \beta)^{2}$ so that the average loss due to relativistic ions equals that due to lighter ions with $\beta$ significantly smaller than one.

As shown in Fig. 4 the $(Z / \beta)^{2}$ values for the ions available at CNAO cover the value of relativistic ions up to $\mathrm{Al}$.

\section{The device under test}

The goal of the beam test is to study the response of a tile of scintillator readout by SiPMs to hadrons impinging on it with the aim of measuring the discriminating power of different $Z / \beta$ and therefore of different relativistic ions. 


\section{Energy loss of ions}
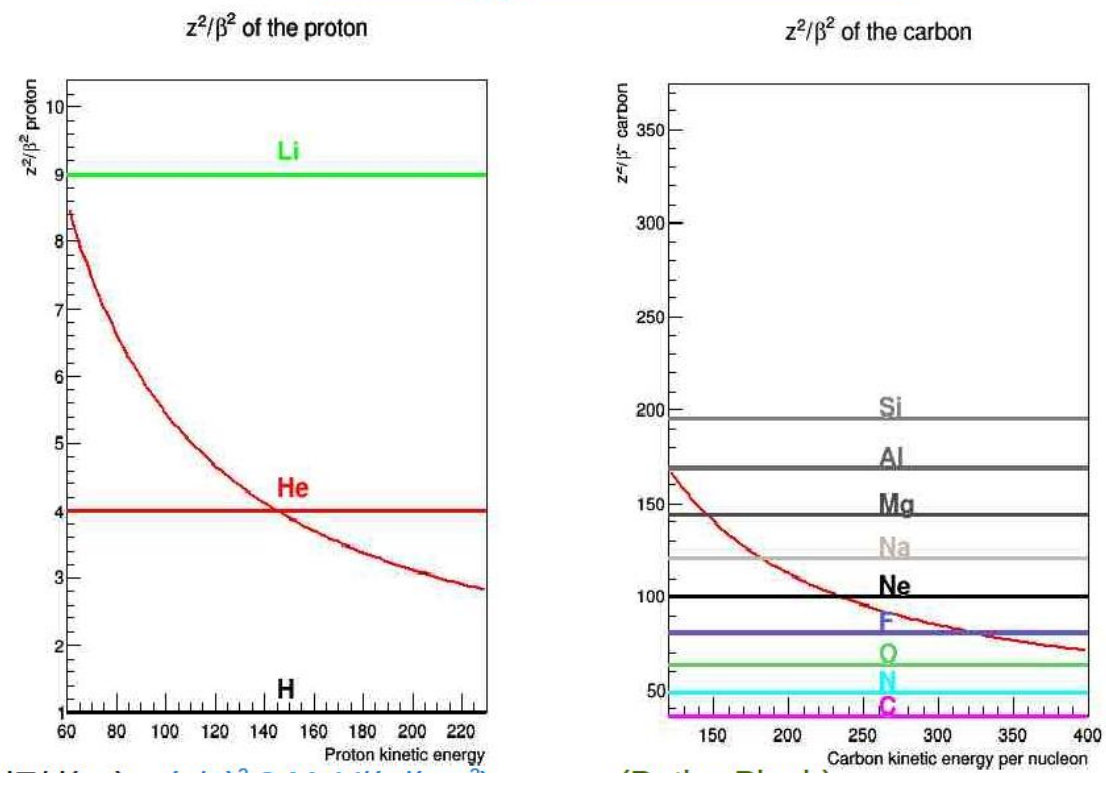

Figure 4. The $(Z / \beta)^{2}$ of protons (left) and carbon ions (right) versus kinetic energy per nucleon compared with that of various relativistic ions.

In order to collect unbiased samples of events we built a setup consisting of two adjacent tiles shown in Fig. 5 (right). The scintillator tile marked as trigger tile is a spare tile of the MEG2 experiment timing counter [2] readout by two opposite sides by $6+6 \mathrm{SiPMs}$ connected in parallel that is used to trigger the data acquisition. The scintillator tile (EJ200) marked as PSD tile is the prototype for the Herd PSD: its size is $10 \times 10 \mathrm{~cm}^{2}$ readout by $3+3$ SiPM from Hamamatsu (S12572) positioned as shown in Fig. 5 (left). The SiPMs on each side are connected in parallel. The two signals (right and left) from the PSD tile and the two signals (right and left) from the MEG2 tile are sent directly to a Tektronix MSO64 oscilloscope with four input channels sampling at $12.5 \mathrm{GHz}$ with 12 bit ADCs. The trigger is the sum of the amplitudes of the two signals from the MEG2 tile that is almost position independent.

\section{Results}

A first set of measurements is the light collected versus the expected energy loss in the tile. The signals from the MEG2 tile are used only for triggering. The relevant quantity is the sum, almost position independent, of the two signals from the HERD tile. Figure 6 reports the distribution of the sum amplitudes for all different beam types and energies.

In Fig.7 the amplitude versus the expected energy loss is displayed. There is no linear relation as naively expected because of saturation of light production in the scintillator at high energy loss rate. The data are fitted very well with the Birks' law [5]

$$
\frac{d L}{d x \rho}=S \frac{\frac{d E}{d x \rho}}{1+k_{\mathrm{B}} \frac{d E}{d x \rho}}
$$



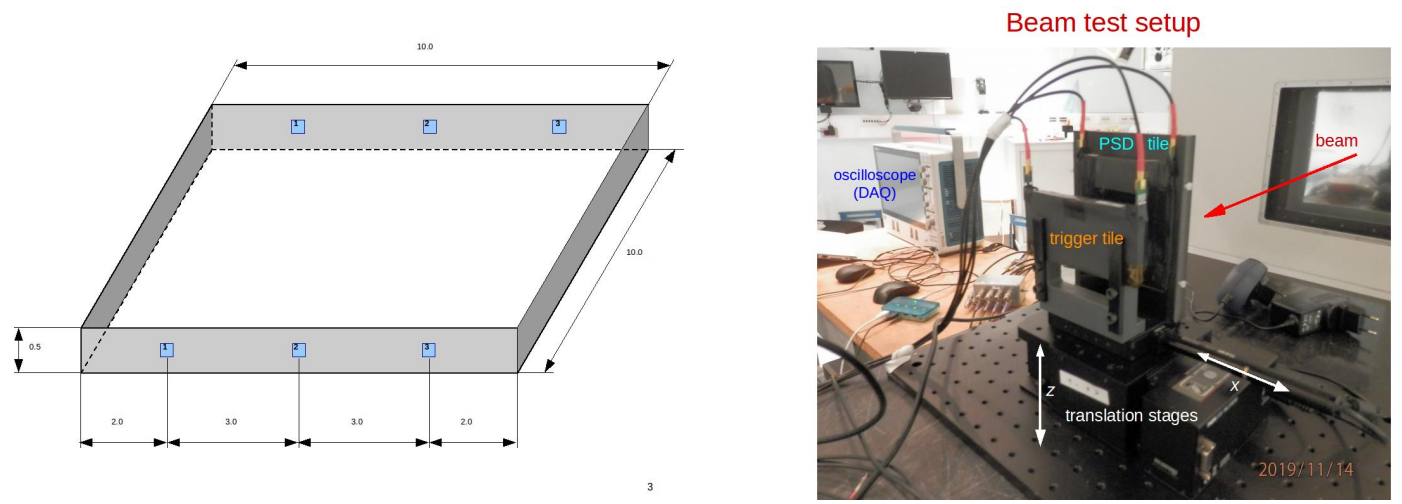

Figure 5. A sketch of the SiPMs positioning on the tile surface (left). A picture of the device under test consisting of two tiles readout by SiPMs (right).

where $k_{\mathrm{B}}$ is a material dependent constant. The result $k_{\mathrm{B}}=12.9 \pm 0.6 \times 10^{-3} \mathrm{~g} / \mathrm{cm}^{2} / \mathrm{MeV}$ is consistent with previous measurements for plastic scintillators [6].

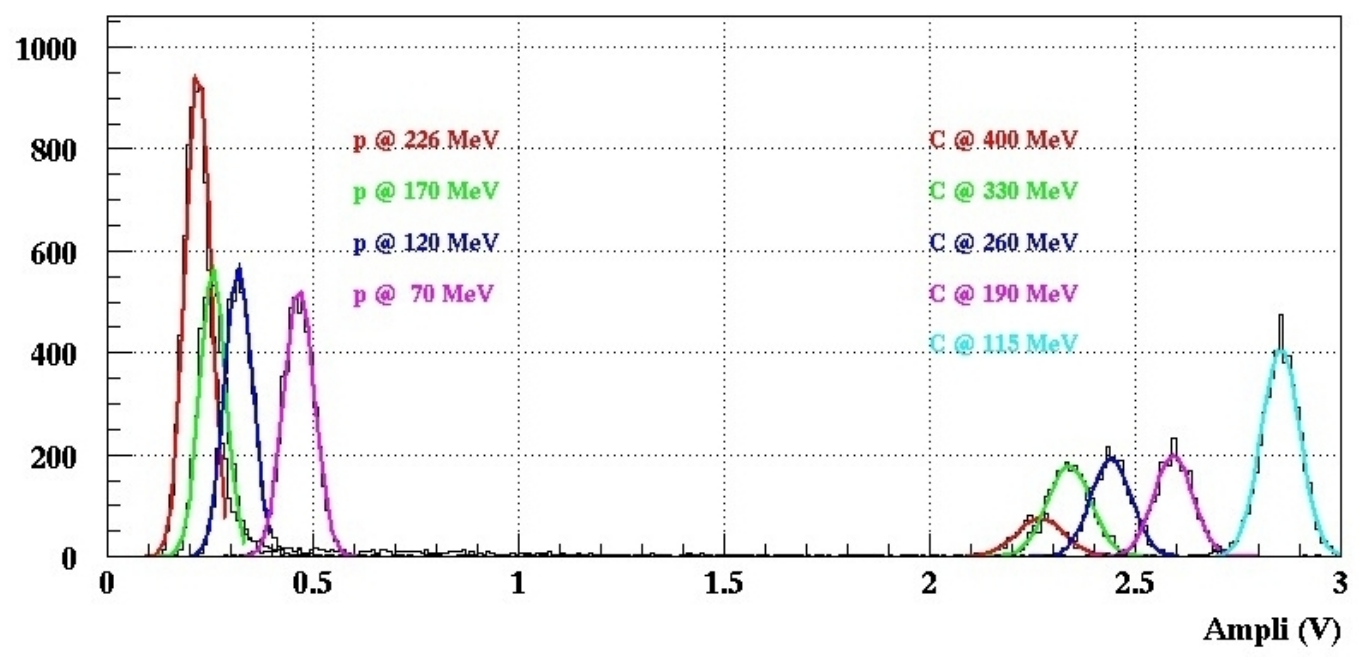

Figure 6. Distribution of SiPM signal amplitudes for the different beam types and energies.

\section{Conclusions}

We present some preliminary results on a beam test with $\mathrm{p}$ and $\mathrm{C}$ ions of a plastic scintillator (EJ200) tile. The test performed at low $\beta$ covers a range of over 60 in expected average energy loss approximately equivalent to the range from $\mathrm{He}$ to $\mathrm{Al}$. The dynamic range in output amplitude is 15 because of saturation effect in production of scintillation light that is well described by Birks' law with a parameter $k_{\mathrm{B}}$ comparable to what measured in the past for similar materials.

\section{Acknowledgments}

We acknowledge the support and cooperation provided by CNAO as the host laboratory. 


\section{Light vs $\mathrm{dE} / \mathrm{dx}$}

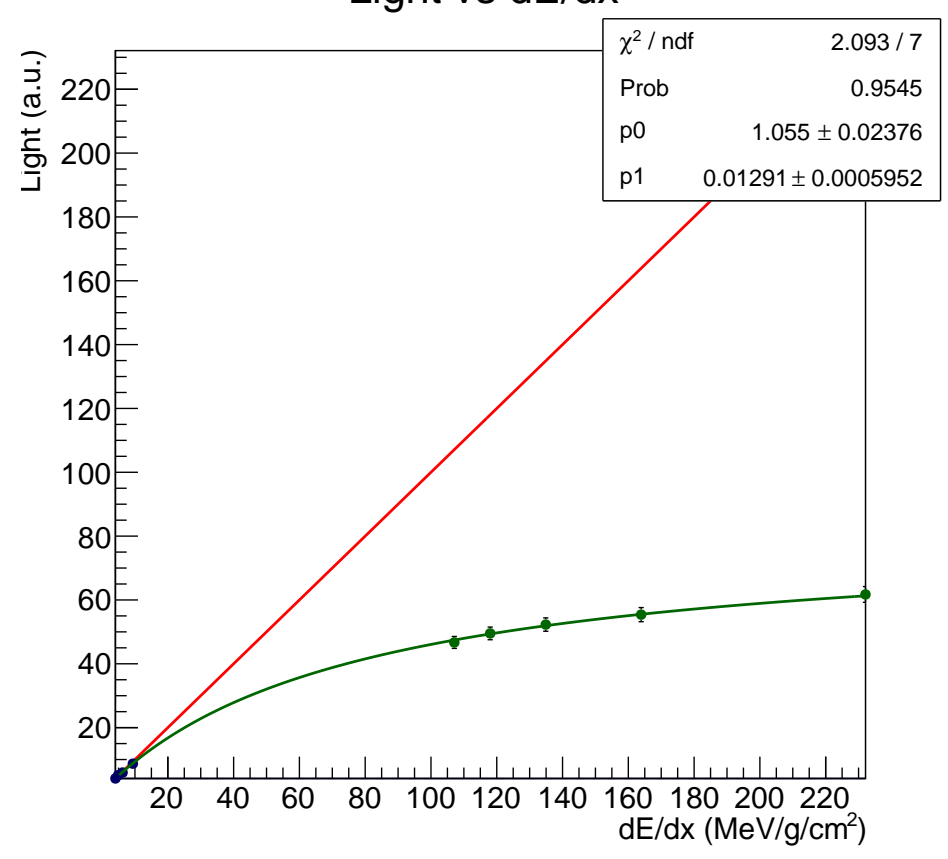

Figure 7. Light output versus the average energy loss. The red line is the expected result in case of exact proportionality between energy loss and light output. Blue (p) and Green (C) axes report the corresponding kinetic energy per nucleon. The green curve shows the result of the data fit with the Birks' formula (see text).

\section{References}

[1] Zhang, Shuang-Nan and others, Introduction to the High Energy cosmic-Radiation Detection (HERD) Facility onboard China's Future Space Station, PoS, 1077, ICRC2017, 2018

[2] P. W. Cattaneo et al., Development and commissioning of the $30 \mathrm{ps}$ time resolution MEG II Pixelated Timing Detector, Nucl. Instr. \& Meth. A 936 (2019) 660, doi.org/10.1016/j.nima.2018.09.055

[3] M. Tanabashi et al. (Particle Data Group), Phys. Rev. D 98, 030001 (2018)

[4] S. Rossi, The National Centre for Oncological Hadrontherapy (CNAO): Status and perspectives, Physica Medica: European Journal of Medical Physics, Volume 31, Issue 4, 333 - 351 (2015), https://fondazionecnao.it/en/

[5] Birks, J.B. (1951). Scintillations from Organic Crystals: Specific Fluorescence and Relative Response to Different Radiations, Proc. Phys. Soc. A 64(10): $874 a ̂ A ̆ S ̧ 877$ (1951) doi:10.1088/0370-1298/64/10/303

[6] Torrisi, L. (2000). "Plastic scintillator investigations for relative dosimetry in proton-therapy". Nucl. Instr. \& Meth. B 170 (3-4) (2000) 523-530 doi:10.1016/S0168-583X(00)00237-8 